\title{
Death among elderly patients in the emergency department: a needs assessment for end-of-life care
}

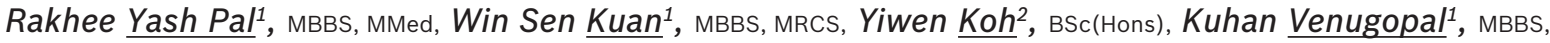 \\ Irwani Ibrahim $^{1}$, MBBS, FRCS
}

INTRODUCTION Elderly patients with serious chronic diseases often present to the emergency department (ED) in the last moments of their life, many with identifiable trajectories of dying: organ failure, advanced cancer and chronic frailty. These patients and their families may benefit more from good end-of-life (EOL) care provision than the standard resuscitative approach. This study aimed to determine the incidence and nature of death among patients aged $\geq 65$ years in an ED, and characterise their trajectories of dying.

METHODS This was a retrospective study carried out over a one-year period in a tertiary ED. All ED deaths in patients aged $\geq 65$ years over this period were included. Information on the patients' demographics, comorbidities and details of death were extracted from the hospital's electronic medical records database. Based on the available information, their Karnofsky Performance Status (KPS) scores and trajectories of dying were ascertained.

RESULTS In one year, 197 patients aged $\geq 65$ years died in the ED, 51.3\% of whom suffered from serious chronic illnesses, with identifiable trajectories of dying. Of these patients, $46.5 \%$ had premorbid functional limitation with KPS scores of $0-40$. However, only $14.9 \%$ of patients had a pre-existing resuscitation status and $74.3 \%$ received aggressive resuscitative measures.

CONCLUSION There is a significant burden of EOL care needs among elderly patients in the ED. Many of these patients have chronic illness trajectories of dying. This study underscores the need for improvement in EOL care provision for dying patients and their families in the ED.

Keywords: death, elderly, emergency department, end-of-life care

\section{INTRODUCTION}

Death is not a rare entity in the emergency department (ED). ${ }^{(1)}$ However, management of death and dying is rarely considered a core aspect of emergency medicine expertise. By nature of their training, most emergency providers are accustomed to dealing with acute and severe illnesses in the ED with a maximally aggressive resuscitation approach. However, this may not be appropriate when managing seriously ill patients with advanced chronic illness trajectories of dying and poor prognosis. ${ }^{(2,3)}$ End-of-life (EOL) care, as part of a palliative approach, may be more suitable in this patient population. ${ }^{(4)}$ Good EOL care acknowledges the multidimensional aspects of dying by providing for the patient's physical, psychological, social and spiritual needs. It focuses on comfort care and holistically addresses the needs of dying patients and their family members. ${ }^{(5)}$ This ensures that these patients receive a good death, one that is in accordance with their wishes and is in keeping with clinical, cultural and ethical standards. ${ }^{(6)}$

Elderly patients with serious chronic diseases often present to the ED in the last moments of their life, many with identifiable trajectories of dying. ${ }^{(7)}$ Three distinct chronic illness trajectories of dying are commonly described: advanced cancer, organ failure and chronic frailty. In patients with advanced cancer, the decline is fairly predictable, with an initial high functional state followed by a sharp deterioration as they enter the terminal phase of their life. In contrast, the trajectory of patients with organ failure is marked by acute exacerbations of illness requiring intensive treatment, with an overall progressive decline in function. Patients with chronic frailty and degenerative neurological diseases often have a low baseline level of function and a protracted course of decline over years. A fourth classical trajectory of dying is that of sudden death in a healthy patient, or one with stable or early chronic illness (Fig. 1). ${ }^{(8)}$ Analysis of trajectories of dying allows for better understanding of a patient's dying process, aids in prognostication and guides EOL decision-making by the physician, as well as the patient and family. ${ }^{(9)}$

Older patients (aged $\geq 65$ years) represent the fastest-growing cohort in the Singapore population. ${ }^{(10)}$ As the population ages and the prevalence of chronic diseases increases, the need for good EOL care is becoming increasingly important. ${ }^{(11)}$ To the best of our knowledge, no studies have been done to characterise the nature of death among this group of patients presenting to the ED, particularly in the Asian population setting. Understanding this burden of care will allow us to identify priorities for improving EOL care in the ED. Hence, this study aimed to determine the incidence and nature of death, as well as the trajectories of dying among patients aged 65 years and above in the ED. Secondarily, it aimed to determine the number of early deaths that occurred in the ward after admission from the ED. 


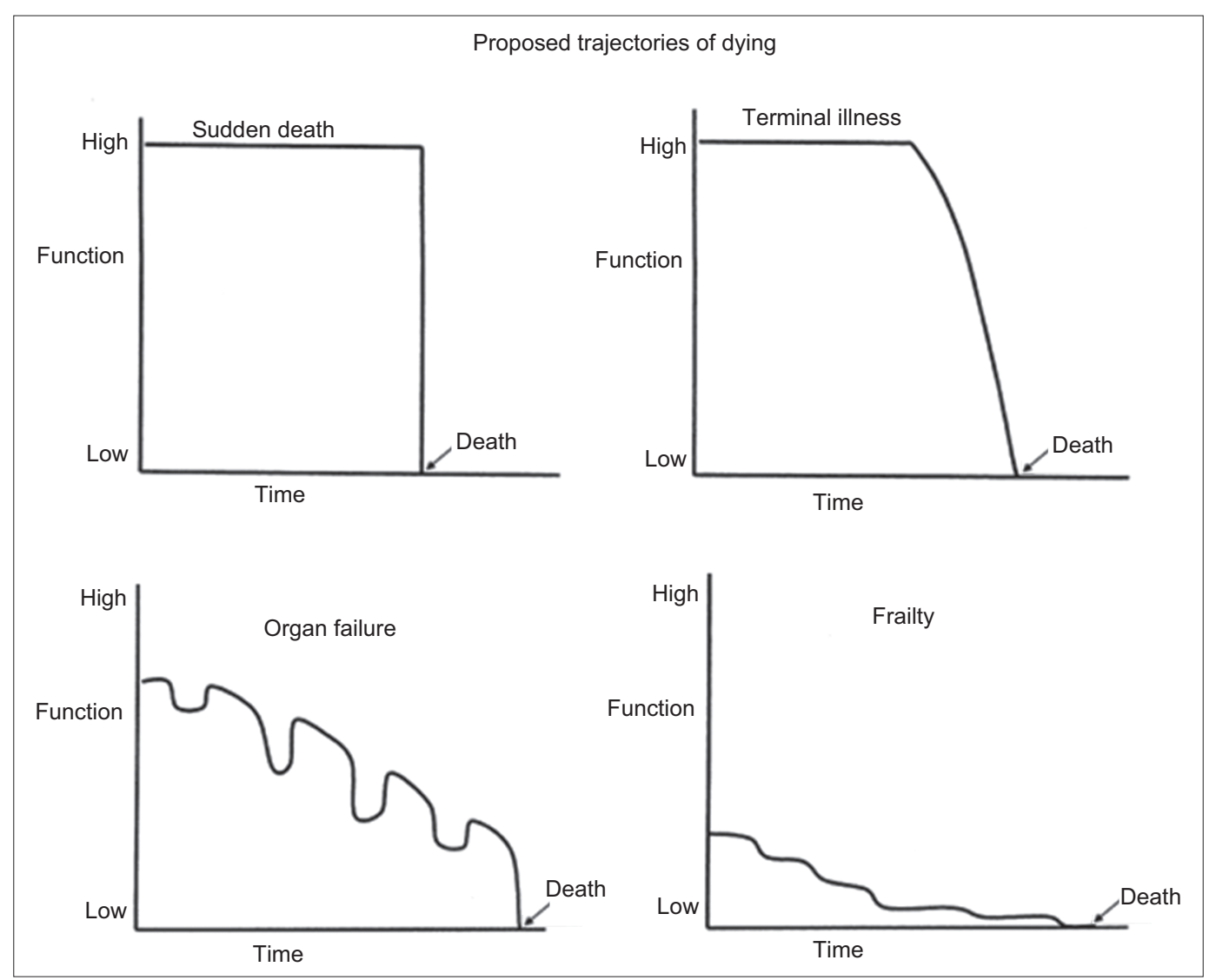

Fig. 1 Image shows four classical trajectories of dying ${ }^{(8)}$ (reproduced with permission from John Wiley and Sons).

\section{METHODS}

This was a retrospective chart review study conducted in the ED at National University Hospital (NUH), Singapore, from 1 January 2011 to 31 December 2011. Approval was obtained from the local institutional review board (DSRB ref. 2013/00214) for waiver of consent. NUH is a tertiary university-affiliated hospital with an estimated 1,100 beds and a yearly ED census of approximately 113,000 patients. All patients aged $\geq 65$ years who died in the ED during the study period were included. Deaths occurring in patients aged $<65$ years or those who had rigor mortis on arrival were excluded. For the secondary analysis, the number of patients who died in the ward within 24 hours of admission from the ED were also reviewed.

Information was extracted from the hospital's electronic medical records database, which would be available to an emergency physician in clinical practice. This included patient demographics, comorbidities, premorbid functional status, clinical presentation, ED interventions, previous resuscitation status documentation or discussion, and details of death (e.g. time and cause of death).

Karnofsky Performance Status (KPS) is an assessment tool intended to assist clinicians in measuring and comparing the functional status of individual patients based on their ability to carry out activities of daily living. The KPS is widely used in clinical practice as well as research, and is a standardised and validated tool regardless of the differences in the personal demographics and clinical conditions of patients. ${ }^{(12,13)}$ While it has limitations of subjectivity, research has shown high interobserver reliability of $0.89 .{ }^{(14)}$ For this study, the patients' KPS scores were assigned based on their documented premorbid functional status. The scores were interpreted as follows: 10-40 - unable to care for self and dependent, with need for institutional care or equivalent; 50-70 - requires varying levels of assistance and unable to work; and 80-100 - fully independent and requires no special assistance. ${ }^{15)}$ 'Score unavailable' meant that we were unable to ascertain the patients' KPS scores, as there was insufficient information in the records.

The trajectories of dying in these patients were classified into one of the following categories: (a) sudden death - patients who were healthy or had stable or early chronic illness; (b) organ failure - patients who had end-stage liver disease, end-stage renal disease, advanced congestive heart failure or advanced chronic obstructive pulmonary disease; (c) chronic frailty - patients who were bed-bound or had cognitive impairment; and (d) advanced cancer - patients who had solid organ tumours with metastatic disease. When a patient had more than one possible trajectory of dying, a hierarchical model was applied in which 'advanced cancer' supplanted 'chronic frailty', which in turn supplanted 'organ failure'. When patients had no known comorbidities or premorbid functional status, they were considered to be well and hence classified under the 'sudden death' trajectory of dying.

Two trained study investigators independently assessed the KPS scores and assigned the trajectories for each patient based on the information derived from the electronic medical records. When there was disagreement on the scores assigned, the case was reviewed by a third investigator. 
All data was entered into a spreadsheet analysis in Microsoft Excel 2013 (Microsoft Inc, Redmond, WA, USA) and subsequently exported into IBM SPSS Statistics for Windows version 22.0 (IBM Corp, Armonk, NY, USA) for analysis. Categorical variables were reported as frequency and percentage values, and continuous data was reported as median (interquartile range [IQR]). Data was analysed using summary statistics.

\section{RESULTS}

In 2011, there were 113,157 ED attendances, of whom $20,595(18.2 \%)$ were patients aged $\geq 65$ years (Table I). Among the 401 deaths pronounced in the ED, 199 (49.6\%) patients were aged $\geq 65$ years. After excluding two deaths that were already in rigor mortis on arrival, 197 patients were included in the final data analysis (Fig. 2). A majority of the deaths analysed were those of men (56.3\%) and ethnic Chinese (75.6\%; Table II). The distribution was consistent with Singapore's ethnic composition. ${ }^{(16)}$ The median age was 78 (IQR 72-84) years.

Analysis of the trajectories of dying of these patients (Fig. 3) showed that $48.7 \%$ were sudden deaths and occurred in those who were healthy or had stable or early chronic illness preceding the ED visit. The remaining $51.3 \%$ of patients had serious chronic illness, with identifiable chronic illness trajectories of dying, namely organ failure, chronic frailty and advanced cancer. The correlation of categorisation of trajectories between study investigators was high, with a kappa value of 0.80 . Three patients with dual trajectories of chronic frailty and organ failure were assigned to the former trajectory according to the hierarchical model for this study.

In the three chronic illness groups ( $\mathrm{n}=101$; Table III), 46.5\% of patients (47/101) had severe premorbid functional limitation with a KPS score of $0-40$, and only $14.9 \%$ (15/101 patients) had a resuscitation status that had been discussed or determined beforehand. A majority of patients $(63.4 \%, 64 / 101)$ with chronic illness trajectories of dying were hospitalised in the six months prior to their death and $74.3 \%$ (75/101) received aggressive resuscitative measures, such as endotracheal intubation, at the time of their death. During the corresponding period, there were 1,163 deaths among patients admitted to the wards from the ED. Of these, $851(73.2 \%)$ were deaths in elderly patients aged $\geq 65$ years. Overall, $146(17.2 \%)$ deaths among elderly patients aged $\geq 65$ years occurred within 24 hours of admission from the ED (Fig. 4).

\section{DISCUSSION}

To our knowledge, this is the first study on the incidence and nature of deaths, particularly the trajectories of dying, among elderly patients presenting to the ED in Singapore. Our results demonstrate that elderly patients who attend the ED for acute terminal crisis events form an important group of users of ED services, and have distinct trajectories of dying and EOL care needs.

In the year 2011, people aged $\geq 65$ years accounted for $10.4 \%$ of Singapore's resident population. ${ }^{(17)}$ However, in our ED, this age group constituted $18.2 \%$ of ED attendances, $37.4 \%$ of admissions, $49.6 \%$ of ED deaths and $73.2 \%$ of deaths in the ward after admission. Overall, in that year, there were 1,564

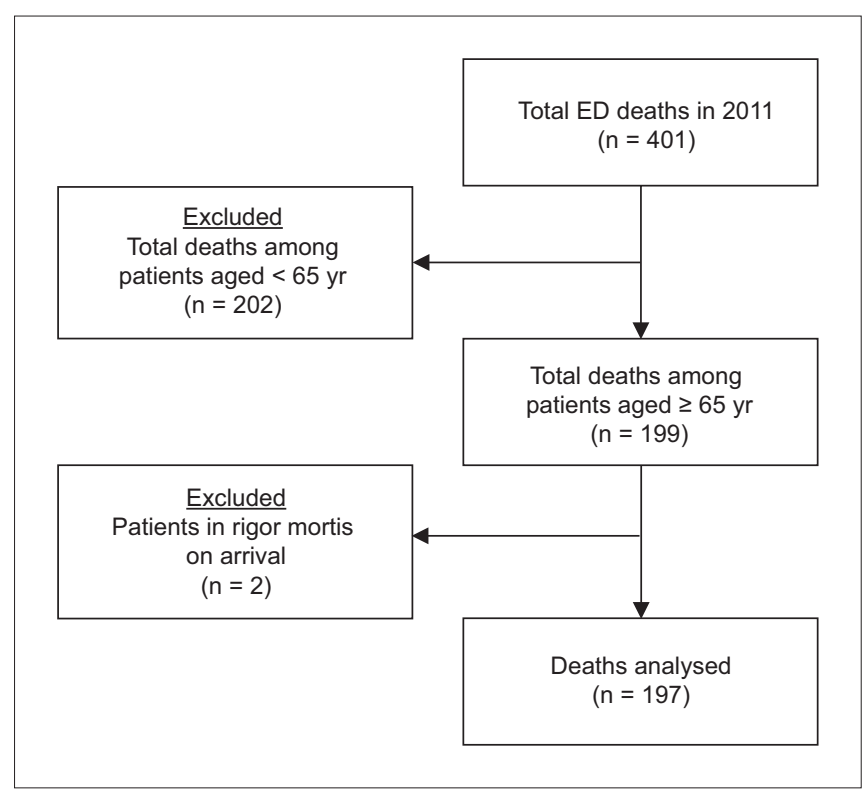

Fig. 2 Flow chart shows patient enrolment. ED: emergency department

Table I. Summary of ED attendances, admissions and deaths.

\begin{tabular}{lcc}
\hline Variable & \multicolumn{2}{c}{ No. (\%) } \\
\cline { 2 - 3 } & Total & Aged $\geq 65$ years \\
\hline ED attendance & 113,157 & $20,595(18.2)$ \\
Admission & 36,400 & $13,617(37.4)$ \\
Death & & \\
$\quad$ Pronounced in ED & 401 & $199(49.6)$ \\
Pronounced in ward & 1,163 & $851(73.2)$ \\
\hline
\end{tabular}

ED: emergency department

Table II. Demographic details and characteristics of analysed deaths in the ED among patients aged $\geq 65$ years $(n=197)$.

\begin{tabular}{lc}
\hline Variable & No. (\%) \\
\hline Age (yr)* & $78(72-84)$ \\
Male gender & $111(56.3)$ \\
Ethnicity & \\
Chinese & $149(75.6)$ \\
Malay & $24(12.2)$ \\
Indian & $14(7.1)$ \\
Other & $10(5.1)$ \\
Cause of death & \\
Heart disease & $127(64.5)$ \\
Pneumonia & $27(13.7)$ \\
Cancer & $12(6.1)$ \\
Chronic pulmonary disease & $9(4.6)$ \\
Aortic aneurysm/dissection & $7(3.6)$ \\
Intracranial haemorrhage & $5(2.5)$ \\
Stroke & $2(1.0)$ \\
Trauma & $2(1.0)$ \\
Other & $6(3.0)$ \\
Referral to coroner & $106(53.8)$ \\
\hline
\end{tabular}

*Data presented as median (interquartile range). †Includes dengue, end-stage renal failure and hepatobiliary sepsis. ED: emergency department

deaths in the hospital after ED attendance, with $25.6 \%$ of deaths (401/1,564 deaths) occurring in the ED itself. Death was a fairly common occurrence in the ED, averaging one death each day 
Table III. Distribution and details of trajectories of dying in four categories.

\begin{tabular}{|c|c|c|c|c|}
\hline \multirow[t]{2}{*}{ Variable } & \multicolumn{4}{|c|}{ No. (\%) } \\
\hline & $\begin{array}{l}\text { Sudden death } \\
\qquad(n=96)\end{array}$ & $\begin{array}{l}\text { Organ failure } \\
\qquad(n=45)\end{array}$ & $\begin{array}{l}\text { Chronic frailty } \\
\qquad(n=43)\end{array}$ & $\begin{array}{l}\text { Advanced cancer } \\
\qquad(n=13)\end{array}$ \\
\hline $80-100$ & $48(50.0)$ & $14(31.1)$ & $0(0)$ & $3(23.1)$ \\
\hline $50-70$ & $13(13.5)$ & $21(46.7)$ & $0(0)$ & $4(30.8)$ \\
\hline $0-40$ & $0(0)$ & $1(2.2)$ & $43(100)$ & $3(23.1)$ \\
\hline Unavailable & $35(36.5)$ & $9(20.0)$ & $0(0)$ & $3(23.1)$ \\
\hline Pre-existing resuscitation status & $3(3.1)$ & $2(4.4)$ & $9(20.9)$ & $4(30.8)$ \\
\hline Hospitalisation in prior $6 \mathrm{mth}$ & $22(22.9)$ & $25(55.6)$ & $29(67.4)$ & $10(76.9)$ \\
\hline Aggressive resuscitation (i.e. intubation) & $89(92.7)$ & $43(95.6)$ & $26(60.5)$ & $6(46.2)$ \\
\hline
\end{tabular}

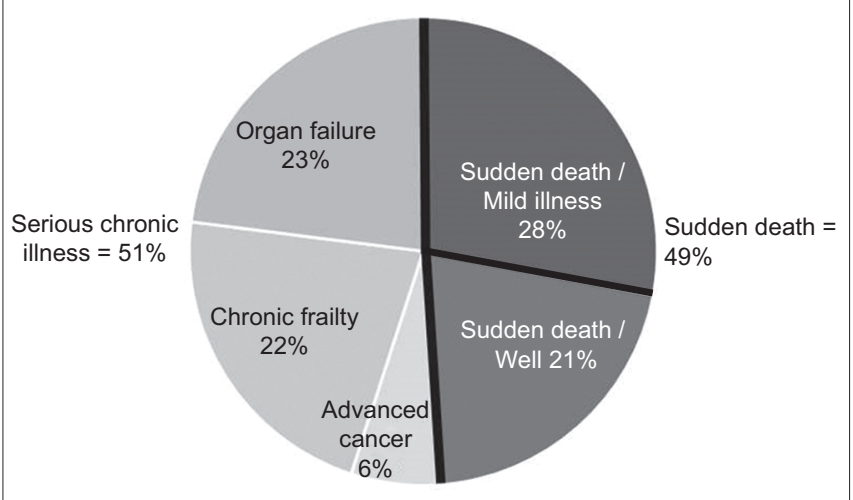

Fig. 3 Pie chart shows the distribution of trajectories of dying in the emergency department among 197 patients aged $\geq 65$ years.

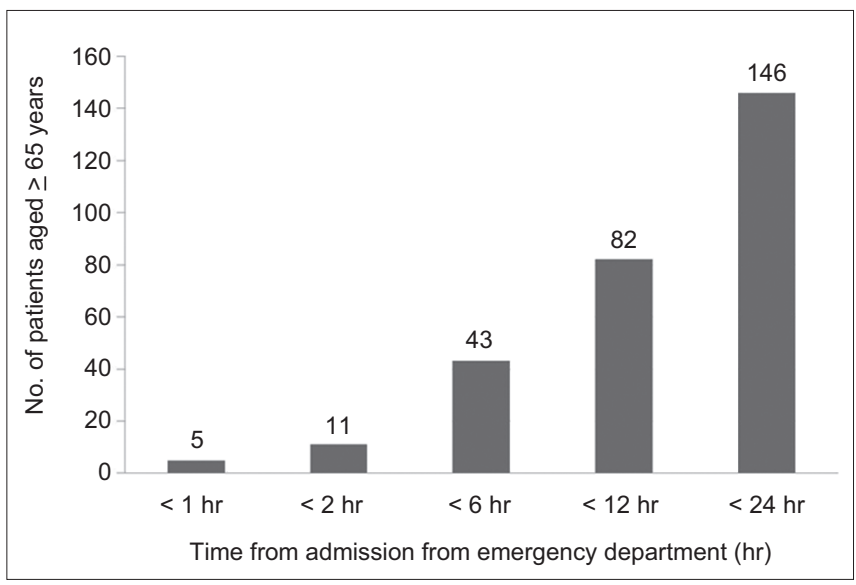

Fig. 4 Cumulative histogram shows deaths in the ward after admission from the emergency department among patients aged $\geq 65$ years.

during the study period. Nearly half of these deaths $(n=199)$ involved elderly patients aged $\geq 65$ years.

In $51.3 \%$ of these elderly patients, there were recognisable chronic illness trajectories of dying. The premorbid functional state of these patients was poor, with $46.5 \%$ of them having a KPS score of $0-40$, indicating that they were mostly bedbound and totally dependent for their activities of daily living. However, only $14.9 \%$ of patients had a pre-established resuscitation status. As a result, most of these patients spent their last moments being aggressively resuscitated in the ED. This was particularly distinct in the chronic frailty trajectory subgroup; there was prior documentation of resuscitation status discussions in only $20.9 \%$
(9/43) of these bedbound patients, although $67.4 \%$ (29/43) of them had been hospitalised in the six months prior to death. More than half of these patients received maximal resuscitation, as suggested by the proportion that was intubated. These findings were similar to an Australian study, which showed that futile measures were often delivered in the ED due to a lack of proper communication and clear documentation about the limits of interventions. ${ }^{(18)}$ This highlights the need for prior discussions on goals of care, to be held by patients' primary physicians and documented in an accessible record. These goals of care can be documented in the form of an advance care plan, which would guide treatment decisions for patients who present acutely to the ED. ${ }^{(18,19)}$

The frequency of death occurring in the ED and the timesensitive nature of these terminal events make it important for emergency physicians to be well versed in prognostication and ethical decision-making skills concerning withholding and withdrawal of life-prolonging measures. Knowledge of the trajectories of dying, premorbid functional status and patients' EOL preferences would facilitate clearer frameworks for clinical decision-making during these difficult times. ${ }^{(3,20,21)}$ Patients with chronic illness trajectories of dying often have complex needs at the end of their lives. ${ }^{(2)}$ This necessitates the application of EOL care principles adapted to emergency medicine practice, such as rapid goals-of-care discussions, ethical medical decision-making, terminal symptom management, a focus on culturally sensitive comfort care and a private space for dignified dying. ${ }^{(18,22)}$ Therefore, EOL care as part of a palliative approach would be more appropriate for these patients than the aggressive resuscitative approach that most ED physicians are accustomed to.

Many EOL discussions have to be done in the ED, necessitating training in communication skills to carry out rapid yet effective discussions with acutely distressed families, with whom no prior rapport has been established. ${ }^{(23)}$ Death is never an easy scenario to deal with, especially in the setting of an acute terminal crisis event, whether in a previously well patient or one with serious chronic illness. Appropriate communication and timely actions on the part of the ED healthcare team can have a significant and positive impact on grieving family members. In this aspect, the sudden death trajectory of dying subgroups may require bereavement support for the families, who are usually unprepared for the suddenness and unexpected nature of the event. ${ }^{(24,25)}$ 
In addition to the 197 deaths that occurred in the ED that year, a further 146 patients aged $\geq 65$ years died in the ward within 24 hours of admission. This represents a large group of imminently dying patients that may have benefitted from early initiation of EOL care in the ED. EOL discussions and decisions on the extent of interventions, if done in the ED, may influence the right siting of subsequent care, be it admission to the intensive care unit, general ward or terminal discharge (discharged home to die) from the ED. Potential advantages to imminently dying patients are the avoidance of unnecessary procedures, shifting of management focus to quality EOL care and being able to spend their last moments with their families. Identification of patients with imminent EOL needs may also allow earlier engagement of the specialist palliative care service, further enhancing the quality of patient care delivery. ${ }^{(9,26)}$

This study was not without limitations. Firstly, it was a singlecentre study and more data may be required from other centres to determine the generalisability of our results to other institutions, particularly non-tertiary institutions. Secondly, applying a hierarchical model for the assignment of a trajectory of dying may represent an oversimplification of a patient's dying process; the boundaries can sometimes be blurred and assignment to a single trajectory can be difficult. However, this was applied for only three patients in the study, in whom there was difficulty in determining whether the trajectory of dying was chronic frailty- or organ failure-related. Notwithstanding, identification of trajectories may serve as a useful approach to prognostication and initiation of goals-of-care discussions in clinical practice. ${ }^{(27)}$ Thirdly, the retrospective nature of the study makes it liable to inherent bias from lack of information, particularly with regard to KPS scores. Future studies that involve formal collection of KPS scores could be incorporated in the documentation of patients who died in the ED.

In conclusion, there is a significant burden of EOL care needs among elderly patients presenting to the ED in the final moments of their lives. Many of these patients have identifiable chronic illness trajectories of dying, although very few had prior EOL discussions and care plans. Our findings indicate that the ED can play an important role in the initiation of EOL discussions. They also demonstrate that the ED is an important site for the provision of EOL care to dying elderly patients and their families. This underscores the need for improving clinical service development, resource allocation, research and communication skills training targeted at the ED for the management of death, dying and bereavement.

\section{REFERENCES}

1. Smith AK, McCarthy E, Weber E, et al. Half of older Americans seen in emergency department in last month of life; most admitted to hospital, and many die there. Health Aff (Millwood) 2012; 31:1277-85.
2. Grudzen CR, Richardson LD, Morrison M, Cho E, Morrison RS. Palliative care needs of seriously ill, older adults presenting to the emergency department. Acad Emerg Med 2010; 17:1253-7.

3. Lamba S. Early goal-directed palliative therapy in the emergency department: a step to move palliative care upstream. J Palliat Med 2009; 12:767.

4. Grudzen CR, Stone SC, Morrison RS. The palliative care model for emergency department patients with advanced illness. J Palliat Med 2011; 14:945-50.

5. Izumi S, Nagae H, Sakurai C, Imamura E. Defining end-of-life care from perspectives of nursing ethics. Nurs Ethics 2012; 19:608-18.

6. Emanuel EJ, Emanuel LL. The promise of a good death. Lancet 1998; 351 Suppl 2:SII21-9.

7. Lunney JR, Lynn J, Foley DJ, Lipson S, Guralnik JM. Patterns of functional decline at the end of life. JAMA 2003; 289:2387-92.

8. Lunney JR, Lynn J, Hogan C. Profiles of older medicare decedents. J Am Geriatr Soc 2002; 50:1108-12.

9. Lamba S, Nagurka R, Murano T, Zalenski RJ, Compton S. Early identification of dying trajectories in emergency department patients: potential impact on hospital care. J Palliat Med 2012; 15:392-5.

10. National Population and Talent Division, Singapore. Issues Paper July 2012 - Our Population Our Future. Available at: https://www.nptd.gov. sg/PORTALS/O/NEWS/ISSUES_P.PDF. Accessed February 24, 2017.

11. Quest TE, Marco CA, Derse AR. Hospice and palliative medicine: new subspecialty, new opportunities. Ann Emerg Med 2009; 54:94-102.

12. Crooks V, Waller S, Smith T, Hahn TJ. The use of the Karnofsky Performance Scale in determining outcomes and risk in geriatric outpatients. J Gerontol 1991; 46:M139-44.

13. Mor V, Laliberte L, Morris JN, Wiemann M. The Karnofsky Performance Status Scale. An examination of its reliability and validity in a research setting. Cancer 1984; 53:2002-7.

14. Schag CC, Heinrich RL, Ganz PA. Karnofsky performance status revisted: reliability, validity, and guidelines. J Clin Oncol 1984; 2:187-93.

15. Karnofsky D, Burchenal J. The clinical evaluation of chemotherapeutic agents in cancer. In: MacLeod C, eds. Evaluation of chemotherapeutic agents. New York: Columbia University Press, 1949: 191-205.

16. Department of Statistics, Singapore. Population Trends 2016. Available at: http://www.singstat.gov.sg/docs/default-source/default-document-library/ publications/publications_and_papers/population_and_population_ structure/population2016.pdf. Accessed February 24, 2017.

17. National Population and Talent Division, Singapore. Population in brief 2012. Available at: https://www.nptd.gov.sg/PORTALS/0/NEWS/ POPULA-4.PDF. Accessed February 24, 2017.

18. Marck $\mathrm{CH}$, Weil J, Lane $\mathrm{H}$, et al. Care of the dying cancer patient in the emergency department: findings from a National survey of Australian emergency department clinicians. Intern Med J 2014; 44:362-8.

19. Brinkman-Stoppelenburg A, Reitjens JA, van der Heide A. The effects of advance care planning on end-of-life care: a systematic review. Palliat Med 2014; 28:1000-25.

20. Murray SA, Kendall M, Boyd K, Sheikh A. Illness trajectories and palliative care. BMJ 2005; 330:1007-11.

21. Chan GK. Trajectories of approaching death in the emergency department: clinician narratives of patient transitions to the end of life. J Pain Symptom Manage 2011; 42:864-81.

22. Bailey CJ, Murphy R, Porock D. Dying cases in emergency places: caring for the dying in emergency departments. Soc Sci Med 2011; 73:1371-7.

23. Smith AK, Fisher J, Schonberg MA, et al. Am I doing the right thing? Provider perspectives on improving palliative care in the emergency department. Ann Emerg Med 2009; 54:86-93, 93.e1.

24. Chan GK. End-of-life models and emergency department care. Acad Emerg Med 2004; 11:79-86.

25. Norton CK, Hobson G, Kulm E. Palliative and end-of-life care in the emergency department: guidelines for nurses. J Emerg Nurs 2011; 37:240-5.

26. Meier DE, Beresford L. Fast response is key to partnering with the emergency department. J Palliat Med 2007; 10:641-5.

27. Mierendorf SM, Gidvani V. Palliative care in the emergency department. Perm J 2014; 18:77-85. 